was remarkable increased in girls with asthma compared to healthy controls $\left(0.49 \pm 0.06 \%\right.$ vs. $0.33 \pm 0.05 \% ; \chi^{2}=3.21$; $\mathrm{gl}=1 ; \mathrm{p}=0.07)$. In children with moderate and severe asthma the functionally compromised genotypes $\mathrm{AA}+\mathrm{AG}$ were identified significantly more frequent comparing to the homozygous normal genotype (GG): $76.9 \%$ vs. $23.1 \%(t=2.3 ; p<0.05)$ and $63.6 \%$ vs. $36.4 \%(t=1.9 ; \mathrm{p}=0.07)$, accordingly. The frequency of A allele was significantly increased in severe asthma cases compared with mild ones $(0.54 \pm 0.1$ vs. $0.30 \pm 0.06 ; \mathrm{gl}=1$; $\left.\chi^{2}=3.5 ; \mathrm{p}=0.054\right)$.

Conclusions The study showed an association of the CC16 A38G polymorphism with more complex and severe forms of asthma. The studied gene was selected because of its important role in regulating inflammatory processes, but it is necessary to conduct further studies of extended range of genes in this ethnic group.

\section{P0-1008 CLINICAL FEATURES OF INCOMPLETE KAWASAKI DISEASE WITH CERVICAL LYMPHADENOPATHY AND FEVER AS INITIAL MANIFESTATIONS}

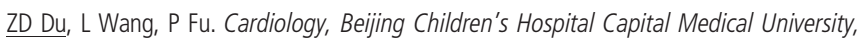
Beijing, China

\subsection{6/archdischild-2014-307384.1625}

Objective This study sought to evaluate the clinical features of the incomplete Kawasaki disease (KD) with cervical lymphadenopathy and fever as initial manifestations.

Methods We conducted a retrospective analysis of the clinical records of consecutive $\mathrm{KD}$ patients admitted to our hospital. Children presenting with cervical lymphadenopathy and fever as the initial manifestations, with none or only one another clinical feature of KD were defined as incomplete KD (IKD) group.

Results A total of 1420 patients were included. Thirty-one $(2.2 \%)$ were classified as IKD. There were no differences in age and gender ratio between two groups. The duration of fever in IKD group $(10.3 \pm 5.7 \mathrm{~d})$ was longer than that in KD group $(6.9 \pm 3.8 \mathrm{~d}, \mathrm{p}<0.05)$. Ten $(32.3 \%)$ children in IKD group presented with bilateral conjunctival congestion. WBC and alanine transaminase (ALT) in IKD group were lower in IKD group. No differences were identified in other laboratory study results between two groups. The rate of non-responders of IVIG in IKD group is lower than KD group (3.3\% vs $18.3 \%)$. Coronary artery lesions in IKD include coronary dilatation in $22(71 \%)$, aneurysm in $5(16.1 \%)$ and giant aneurysm in $3(9.7 \%)$, which is higher than $\mathrm{KD}$ group $(27.7 \%, 5.2 \%$ and $1.1 \%)$.

Conclusions Children with incomplete KD and cervical lymphadenopathy and fever as initial manifestations presented with longer period of fever and higher incidence of coronary artery aneurysm. Thus awareness of IKD should be kept in mind in this special group of children.

\section{PO-1009 WITHDRAWN}

\section{P0-1010 ACUTE OTITIS MEDIA - A RELIABLE WARNING SIGN FOR PRIMARY IMMUNODEFICIENCIES?- A CRITICAL APPRAISAL}

F Furtado, A Cordeiro, JF Neves, C Neves. Primary Immunodeficiencies Unit - Pediatric Department, Hospital Dona Estefania - CHLC, Lisboa, Portugal
Background and aims Acute otitis media (AOM) is the most common infection in childhood, resulting from both anatomic and immunologic specificities of this age group. Recurrent AOM has been defined as one of the warning signs for primary immunodeficiencies (PID), In this study we evaluated the strength of recurrent AOM as clinical predictor of PID.

Methods Retrospective study (August 2010-December 2013) which included all patients referred to PID appointment because of recurrent AOM ( $\geq 8$ AOM episodes/year). Syndromic patients or those presenting with another warning sign for PID were excluded. Clinical, demographic and laboratory results were analysed and statistical analysis was made using SPSS 20.

Results Seventy-five patients were included (median age 37,8 months; $62,7 \%$ male gender), corresponding to $15 \%$ of all first appointments. Other comorbidities were present in $20 \%$ of the patients and $17 \%$ had ORL surgery prior to PID referral. In most patients, the immunologic screening consisted on the evaluation of humoral function, but in selected cases other studies were performed (namely complement and lymphocyte immunophenotyping).

A PID was identified in 12 children (16,0\%) and the majority of these patients had other distinctive feature (personal or familiar antecedent of infection or auto-immunity, 66,7\%, $\mathrm{p}<0,05$ ). Nine children $(12,0 \%)$ underwent prophylactic cotrimoxazole. The average length of follow-up was 11,2 months.

Conclusion Despite being a very frequent cause of immunologic screening, in this study recurrent $\mathrm{AOM}$ was not found to be a good predictor of underlying PID, unless the patients presents other significant personal or family history.

\section{PO-1011 HIGH RATE OF SCHOOL ABSENTEEISM AND DROP-OUT IN CHILDREN WITH JUVENILE IDIOPATHIC ARTHRITIS FROM A DEVELOPING COUNTRY}

${ }^{1} \mathrm{R}$ Gulati, 'K Vijayasekharan, ${ }^{2}$ VS Negi. ${ }^{1}$ Pediatrics, Jipmer, Pondicherry, India; ${ }^{2}$ Clinical Immulology, Jipmer, Pondicherry, India

\subsection{6/archdischild-2014-307384.1627}

Background and aims Juvenile idiopathic arthritis (JIA) causes significant physical and functional disability. Children miss school due to illness, multiple hospital visits or admissions. Frequent absence from school is consistently reported to adversely affect academic performance. Our aim was to assess the rate of school absenteeism and drop out among Indian patients of JIA.

Methods The study was carried out on 69 children, 32 female, 37 male. The children and their parents were interviewed for details of school attendance, number of days and frequency of absence from school. Medical records were examined for hospital admissions and follow-up visits to supplement the information obtained from families.

Results The median number of school days missed/year were 41 (4-300; IQR- 20,120), representing $21.5 \%$ of school days.

\begin{tabular}{lll} 
Abstract PO-1011 Table 1 & School absenteeism - JIA subtypes \\
\hline & School days lost/year \\
JIA subtype & Mean \pm SD & ${ }^{*} \mathrm{p}$ \\
Oligoarticular & $61.87 \pm 97.34$ & \\
Polyarticular RF-ve & $46.35 \pm 56.28$ \\
Polyarticular RF+ve & $75.57 \pm 83.39$ \\
SoJlA & $112.21 \pm 75.12$ \\
& $51.84 \pm 40.27^{\star}$ Kruskal Wallis test \\
ERA & & 0.018 \\
\hline
\end{tabular}

